\title{
Avaliação funcional de idosos em três programas de atividade física: caminhada, hidroginástica e Lian Gong
}

\author{
Marcelo E.S. Nunes \\ Suely Santos
}

https://doi.org/10.5628/rpcd.09.02-03.150

\author{
Laboratório de Comportamento Motor (Lacom) \\ Escola de Educação Física e Esporte \\ Universidade de São Paulo \\ Brasil
}

\begin{abstract}
RESUMO
Os benefícios que estão associados com a actividade física regular proporcionam uma vida independente, melhora na qualidade de vida e capacidade funcional durante o envelhecimento. $\mathrm{O}$ objectivo desse estudo foi comparar três programas de actividade física, especificamente, Caminhada, Hidroginástica e Lian Gong, a partir da capacidade funcional dos praticantes. A capacidade funcional foi avaliada através da Bateria de Testes de Fullerton. Participaram deste estudo 113 indivíduos divididos em três grupos: Caminhada $(n=38)$, Hidroginástica $(n=38)$ e Lian Gong ( $\mathrm{n}=37)$, com idade entre 60 e 84 anos. A análise dos resultados revelou que o grupo Caminhada foi superior nos testes de força de membros inferiores e capacidade aeróbia, comparado com a Hidroginástica e o Lian Gong. Através do teste que avalia agilidade e equilíbrio dinâmico, detectou-se uma inferioridade do grupo Lian Gong em relação aos outros dois grupos. Em relação ao teste que avalia força de membros superiores, o grupo Hidroginástica foi superior aos demais grupos. Mesmo considerando a ausência de um grupo controle, pode-se concluir que os programas de actividade física avaliados apresentam benefícios para a capacidade funcional e, consequentemente, à saúde das idosas. Além disso, pode-se afirmar que a Caminhada e a Hidroginástica se complementam na manutenção das capacidades motoras de idosos.
\end{abstract}

Palavras-chave: capacidade funcional, idoso, actividade física

\begin{abstract}
Functional evaluation of elderly people in three physical activity programs: walking, water therapy exercise, and Lian Gong

The benefits that are associated with regular physical activity optimize health, providing an independent life, quality of life improvement and to functional capacity increase during aging. The purpose of this study was to compare three physical activity programs, specifically, Walking, Water Therapy Exercise, and Lian Gong, based on the functional capacity tests. Functional capacity was evaluated using the battery of tests of Fullerton. One hundred thirteen individuals were assigned in three groups: Walking $(N=38)$, Water Therapy Exercise $(n=38)$, and Lian Gong $(n=37)$, from 60 to 84 years old. Statistical analyses revealed that Walking group was superior in lower body strength and aerobic capacity tests, in comparison to Water Therapy Exercise and Lian Gong groups. Agility and dynamic balance test showed that Lian Gong group was inferior to both other groups. Water Therapy Exercise group was superior in upper body strength. Even considering the absence of a control group, it can be concluded that the physical activity programs studied show benefits to the functional capacity and, consequently, to the health of the elderly women. Additionally, Walking and Water Therapy Exercises groups seemed to be complementary in maintaining motor capacities in the elderly.
\end{abstract}

Key-words: functional capacity, elderly, physical activity 


\section{INTRODUÇÃO}

Os estereótipos associados ao envelhecimento têm sido revistos ${ }^{(8)}$, o que transpassa a ideia de que os estágios mais avançados da vida podem ser momentos propícios para novas conquistas pessoais. Isto se justifica pelos estudos demográficos sobre a distribuição populacional mundial realizados nas últimas décadas ${ }^{(12)}$, ou seja, essas tendências populacionais vêm se confirmando na medida que há um aumento do segmento da população acima de 65 anos de idade e esse ritmo de crescimento deve prolongar-se ainda pelos próximos anos. O crescimento acentuado desse segmento populacional é o resultado do que os pesquisadores chamam de transição demográfica(13). O processo de transição demográfica refere-se ao efeito das taxas de mortalidade e nascimento sobre o tamanho e distribuição etária de populações. O ritmo da transição demográfica varia de país para país e mesmo, de região para região dentro do mesmo país(23), e é causado por um conjunto complexo de factores socioeconómicos.

Consequentemente, é notável a recente proliferação de programas e serviços voltados às pessoas idosas, que é um indicativo de que a sociedade está mais sensível às demandas do envelhecimento populacional. Contudo, como alerta Debert ${ }^{(8)}$, o sucesso surpreendente dessas iniciativas é proporcional à precariedade dos mecanismos de que dispomos para lidar com a velhice avançada nas mais diversas áreas. Os acalorados debates sobre previdência social, por exemplo, ilustram esse quadro e, no meio académico, mesmo considerando a elevação do número de informações geradas relativa ao processo de envelhecimento, o entendimento sobre o controle de acções motoras (por exemplo) nessa etapa da vida ainda é incipiente.

Muitos idosos mantêm um nível de funcionamento perigosamente próximo de suas capacidades máximas para desempenhar actividades triviais(21).

Actividades físicas diárias como caminhar, subir escadas, levantar de uma cadeira e manter a casa podem representar $80 \%$ do VO2 máximo em pessoas idosas sedentárias ${ }^{(7)}$. Dessa forma, qualquer pequena alteração ou declínio de ordem física poderia levar o indivíduo idoso a torna-se dependente e/ou incapacitado para determinada tarefa cotidiana.

O número de pessoas idosas participantes de algum programa de actividade física é cada vez maior. Esse tipo de comportamento parece sensato já que, segundo Chandler \& Hadley(7), a actividade física é a chave para promover uma boa saúde e uma melhor qualidade de vida e a prática regular traz muitos benefícios para a saúde e previne certas doenças ${ }^{(4)}$. Spirduso(22) afirma que a saúde e a actividade física juntas têm grande probabilidade de contribuir para a diminuição da mortalidade nesta população, melhorando os hábitos e ocasionando consequentemente uma melhora física, mental e emocional, pois a participação em um programa regular de actividade física previne, auxilia e traz muitos benefícios durante o envelhecimento destas pessoas.

A participação em um programa cujo conteúdo envolve exercícios aeróbicos ou de força colaboram com respostas favoráveis para um envelhecimento saudável, ou seja, um programa de actividade física contribui para a intervenção e/ou redução de inúmeros declínios funcionais ocorridos com o envelhecimento, além da redução de factores de riscos associados com algumas doenças crónico-degenerativas. Os benefícios que estão associados com a actividade física regular optimizam uma melhora na saúde, proporcionando uma vida independente, melhora na qualidade de vida e na capacidade funcional das pessoas idosas ${ }^{(1)}$.

Capacidade funcional é definida como ter a capacidade fisiológica para realizar normalmente actividades diárias de maneira segura, sem que haja uma fadiga indevida(19), durante a solicitação de alguns componentes como: capacidade aeróbica, força muscular, flexibilidade, equilíbrio, destreza manual, etc.(15). A avaliação da capacidade funcional de indivíduos idosos torna-se importante, na medida em que, a partir dela, é possível encontrar meios de prevenir ou retardar o início de fragilidades físicas que ocorrem em idades avançadas ${ }^{(6)}$. Com esse tipo de avaliação, há o acesso a atributos fisiológicos (força, capacidade aeróbia, flexibilidade, agilidade, equilíbrio), que são solicitados em actividades executadas no dia-a-dia( ${ }^{(18)}$. A identificação de um ou mais déficits nestes atributos fisiológicos significa um planejamento mais adequado para o programa de actividade física(20). Cech e Martin(6) enfatizam, inclusive, que a avaliação da capacidade funcional é a maneira mais adequada para observar os programas de actividade 
física e suas relações com habilidades relacionadas ao trabalho, lazer e actividades básicas diárias. Muitos protocolos tradicionais utilizados para avaliar as capacidades motoras (teste de esteira ou ciclo ergômetro, teste de 1 RM de força, etc.) de certo modo foram desenvolvidos e validados para serem utilizados por pessoas jovens e, portanto, geralmente inapropriados para serem utilizados com idosos. Além disso, esses protocolos requerem equipamentos caros e certa experiência e técnica para a participação no teste, tornando-se impraticável fora de uma clínica especializa$\mathrm{da}{ }^{(18)}$. Ademais, alguns protocolos se preocupam primariamente em detectar a limitação física, dificultando assim a correlação com a realização de actividades específicas diárias como tomar banho, trocar de roupa, caminhar, agachar ou subir escadas ${ }^{(9)}$.

Embora a capacidade funcional seja vital e também tenha sérias implicações para o sistema de saúde, em geral, idosos só recebem atenção quando eles apresentam disfunções. Através da avaliação das capacidades funcionais de um indivíduo idoso, poderiam ser identificadas possíveis fragilidades físicas que ocorrem em idades avançadas e, desta forma, prevenir e reduzir uma série de declínios funcionais ocorridos com o envelhecimento, proporcionando uma melhora na capacidade funcional e na qualidade de vida das pessoas idosas ${ }^{(19)}$.

Assim, com o intuito de avaliar o impacto na capacidade funcional de idosos de diferentes programas de actividade física, foi realizada uma análise comparativa de um programa de Hidroginástica, de Caminhada e o Lian Gong, através da bateria de testes de Fullerton (18, 20).

\section{MÉTODO}

\section{Amostra}

Para este estudo, os indivíduos foram seleccionados através de convites feitos em academias, clubes, parques municipais, praças de esportes, e etc. Os critérios de inclusão foram: (a) ser do sexo feminino; (b) ter idade entre 60 e 84 anos; (c) participar de programas de Hidroginástica, Caminhada e Lian Gong há pelo menos três anos; e (d) frequentar as aulas no mínimo duas vezes por semana. Foram seleccionados 38 praticantes de hidroginástica, 38 de caminhada e 37 de Lian Gong completando um total de 113 sujeitos na pesquisa.
Todos os participantes assinaram um Termo de Consentimento Livre e Esclarecido, contendo todas as informações sobre os testes (riscos, benefícios, aplicabilidades e outros) e informações sobre a utilização dos dados em eventos científicos, produções e publicações científicas. Em todos os momentos da pesquisa foi mantido sigilo sobre a identidade dos participantes.

Os programas de actividade física de hidroginástica, caminhada e Lian Gong foram seleccionados por serem populares entre os idosos de determinadas regiões da cidade de São Paulo. Uma breve descrição desses programas é apresentada a seguir:

\section{Hidroginástica}

A Hidroginástica surgiu no final da década de 1980, com o objectivo de utilizar exercícios aquáticos na posição vertical não mais visando a reabilitação e sim o condicionamento físico. Com o passar do tempo, a hidroginástica criou uma identidade própria e evoluiu para um sistema de condicionamento físico que pode ser utilizado não só pelas populações sedentárias com limitações e restrições, mas também por atletas de diversas modalidades esportivas, que encontram nesta modalidade uma eficiente forma de recuperação ativa e de substituição de parte do treinamento cardiovascular(16). A hidroginástica se diferencia de outras actividades, realçando alguns benefícios, devido às propriedades físicas que o meio líquido oferece(5).

\section{Caminhada}

Caminhar constitui uma das mais naturais actividades físicas por ser uma actividade simples, facilmente controlável, sem a necessidade de equipamentos especiais e pode ser praticada por qualquer pessoa. Na caminhada o movimento básico é a marcha, que se diferencia da corrida, pois na marcha não existe a fase de vôo, momento em que os pés deixam de ter contacto com o solo. Além de ser considerada uma das práticas mais seguras do ponto de vista cardiovascular e ortopédico, apresenta maior índice de aderência em exercícios para prevenção de problemas e promoção da saúde(14). A prática de caminhada não necessita de equipamentos especiais, podendo ser praticada em qualquer ambiente, próximo de casa ou do trabalho, além de poder ser praticada de forma individual ou colectiva. 


\section{Lian Gong}

A ginástica Lian Gong foi desenvolvida na China, pelo Dr. Zhuang Yuen Ming, médico ortopedista da Tradicional Medicina Chinesa (TMC), na década de 60. O Lian Gong (pronuncia-se Lian Kung) é uma técnica que une a medicina terapêutica chinesa e a cultura física, que para os chineses significa: o fortalecimento harmonioso do corpo, permitindo o pleno funcionamento e utilização dos músculos, tendões e ossos. O Lian Gong é descrita como uma ginástica terapêutica preventiva que começou a ser divulgada desde 1975 e, provavelmente por ser composta de movimentos de simples execução, ganha a cada dia um grande número de praticantes.

De acordo com Ming(17), o Lian Gong compõe-se de duas partes: a primeira trata da prevenção e tratamento de dores no pescoço, ombros, costas, região lombar, glúteos e pernas; a segunda trata da prevenção e tratamento de dores nas articulações dos membros superiores e inferiores, de tenossinovites e desordens funcionais dos órgãos. Ming(17) ressalta que o Lian Gong é considerado pelo Conselho Nacional e pelo Conselho de Práticas Corporais de Shangai como uma das técnicas que mais bem representa a cultura milenar chinesa na área de práticas corporais dentro e fora da China.

O Lian Gong consiste na execução de seis séries de exercícios com a duração de 24 minutos. Os exercícios são simples, acessíveis a pessoas de todas as idades e podem ser realizados em qualquer lugar sem necessidade de roupas especiais ou de equipamentos.

\section{Medidas}

\section{Capacidade funcional}

Rikli \& Jones $(18,19)$ desenvolveram e validaram uma bateria de testes de aptidão funcional para o Ruby Gerontology Center, na California State University (também conhecidos por "Fullerton Tests") que foram definidos como testes que avaliam a capacidade fisiológica para desempenhar actividades normais do dia-adia de forma segura e independente, sem que haja uma fadiga indevida. Cada um dos atributos fisiológicos avaliados, os quais dão suporte aos comportamentos necessários para desempenhar tarefas diárias, são: força de membros superiores e inferiores, capacidade aeróbia, flexibilidade de membros supe- riores e inferiores, e agilidade motora/equilíbrio dinâmico. Adicionalmente, as autoras também utilizaram o índice de massa corporal (IMC) como uma estimativa da composição corporal. Os testes foram especificamente idealizados para uso num ambiente de campo e/ou clínico (nonlaboratory), particularmente, para serem capazes de fornecer medidas escalares contínuas através de uma ampla faixa de níveis de habilidade que são tipicamente encontrados na população idosa em geral. A limitação dos testes de medida de performance física desenvolvidos anteriormente, é que eram apropriados para populações específicas de indivíduos, ora para idosos de saúde mais frágil, ora para idosos altamente funcionais, o que dificulta a comparação de dados de diferentes faixas de idade e níveis de habilidade.

Assim, os participantes do estudo realizaram os seguintes testes:

\section{1) Levantar da Cadeira (30s Chair Stand)}

Proposta: Avaliar força de membros inferiores. Protocolo: O teste inicia com o participante sentado no meio da cadeira, com o corpo erecto e os pés apoiados no chão. Os braços deverão estar cruzados junto ao peito com as mãos apoiadas nos ombros. Ao sinal de "valendo" o participante deverá levantarse completamente e retornar a posição inicial. O participante será motivado a completar o movimento o maior número de vezes possível durante 30 segundos. Após a demonstração do teste e uma pratica de três repetições, ele será convidado a iniciar o teste propriamente dito.

\section{2) Rosca (Arm Curl)}

Proposta: Avaliar força de membros inferiores. Protocolo: $\mathrm{O}$ avaliado deverá estar sentado na cadeira com o tronco erecto e com os pés apoiados no chão. O participante escolherá o lado do corpo em que realizará a avaliação, o teste deverá iniciar com o peso perpendicular ao solo, com os braços estendidos. Após o sinal de "valendo" o avaliado deverá realizar uma flexão de cotovelo, sem lançar o mesmo à frente, executando o maior número de repetições possíveis durante 30 segundos. Após a demonstração do teste e uma prática de três repetições com o lado escolhido ele será convidado a iniciar o teste. 


\section{3) Caminhar 6 Minutos (6-Minute Walk)}

Proposta: Avaliar resistência aeróbia. Protocolo: Ao sinal de "valendo" os participantes iniciarão uma caminhada o mais rápido possível, sem correr, em um percurso retangular de $45,72 \mathrm{~m}$ demarcado por cones a uma distância de $4,57 \mathrm{~m}$ entre cada um. Essa caminhada será realizada durante seis minutos sendo contabilizado o número de voltas realizadas pelo participante e ao término do tempo, o avaliado deverá parar por alguns segundos no local onde está para identificar quantas marcas percorreu na última volta, após a identificação realizará mais uma volta completa com intuito de recuperar-se. Os números de voltas e marcas percorridas serão convertidos em metros, determinando a distância percorrida durante o teste.

\section{4) Sentar e Alcançar (Chair Sit-and-Reach)}

Proposta: Avaliar flexibilidade de membros inferiores. Protocolo: O teste inicia com o participante sentado na cadeira. Uma das pernas deverá estar com joelho flexionado em aproximadamente $90^{\circ}$ e o pé apoiado no chão, enquanto a outra perna deverá estar estendida. Com os braços estendidos e os dedos médios um sobre o outro, o participante deverá realizar uma flexão de quadril sobre a perna estendida atingindo o máximo que conseguir e manter-se na posição por dois segundos enquanto o avaliador faz a medida. Esta medida será a distancia entre os dedos médios e a ponta do pé, sendo considerado negativo anterior à ponta do pé e positivo a distância em que os dedos passarem da ponta do pé, assumindo como marco zero a ponta do pé, essa medida será feita em centímetros. O avaliado escolherá o lado do corpo em que será feito o teste e terá direito a duas tentativas marcando o seu melhor resultado.

\section{5) Mãos nas Costas (Back Scratch)}

Proposta: Avaliar a flexibilidade de membros superiores. Protocolo: O teste iniciará com o participante em pé e o mesmo deverá tocar suas costas com uma das mãos por cima do ombro e com a palma da mão voltada para as costas, enquanto com a outra mão sob o ombro e com a palma da mão voltada para fora, ele deverá tentar aproximar os dedos médios. $\mathrm{O}$ avaliador deverá medir no ponto onde o avaliado ficar imóvel a distância entre os dedos médios, adotando como marco zero a junção das pontas dos dedos, sendo considerado positivo a distância que o avaliado conseguir ultrapassar o marco zero e negativo a distância que faltar para a junção dos dedos. Essa medida será feita em centímetros. O avaliado escolherá o lado do corpo em que será feito o teste e terá direito a duas tentativas marcando o seu melhor resultado.

6) Levantar, Ir e Voltar (8-Foot Up-and-Go) Proposta: Avaliar agilidade e equilíbrio dinâmico. Protocolo: $\mathrm{O}$ avaliado iniciará o teste sentado no meio da cadeira, em posição ereta, com os pés apoiados no chão e as mãos na coxa. Ao sinal de "valendo" o participante levantará da cadeira e caminhará rapidamente (sem correr) e dará a volta em um cone que estará a uma distância de $2,44 \mathrm{~m}$ da cadeira, e deverá voltar a posição inicial. Ao dar o sinal de partida o avaliador deverá iniciar o cronômetro e só parar o mesmo quando o avaliado estiver novamente na posição inicial. Após a demonstração e uma tentativa de pratica o avaliado terá direito a duas tentativas anotando-se o melhor resultado.

\section{Índice de massa corporal (IMC)}

O Índice de Massa Corporal (IMC), recomendado pela Organização Mundial de Saúde, tornou-se uma das maneiras mais utilizadas para a avaliação do peso corporal de adultos. Apesar de não discriminar os componentes gordo e magro da massa corporal total, o IMC é o método mais utilizado para avaliação do grau de risco associado à obesidade. O IMC é calculado dividindo-se o peso em Kilogramas (Kg) pela Altura (m) elevada ao quadrado.

\section{Nível de Actividade Física Diária}

Em pesquisas epidemiológicas, questionários de actividades físicas têm sido utilizados para se ter acesso a quantidade de actividade física habitual dos entrevistados(24), sendo importante por considerar não somente o gasto energético total durante um certo número de dias ou semanas, mas também para considerar o tipo de actividade física habitual durante um período(2).

Neste estudo, foi utilizado um questionário baseado no Questionário de Atividade Física de Beacke(2,3) 
A

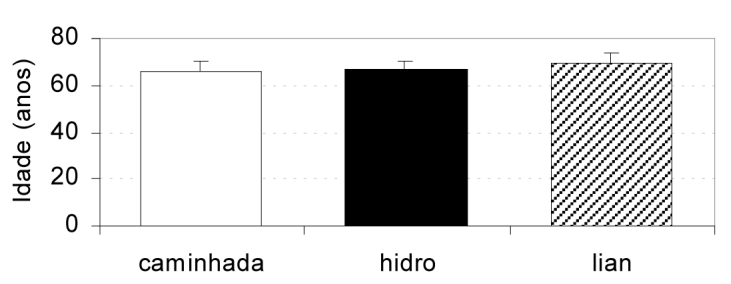

C

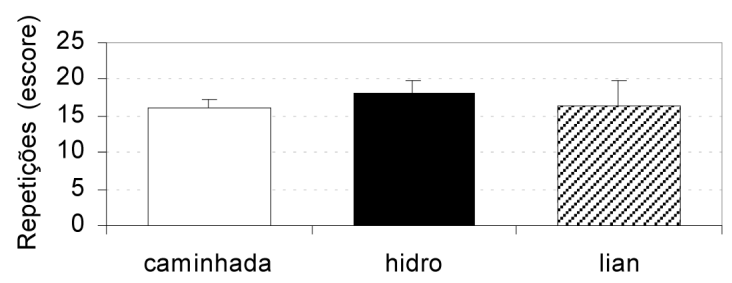

E

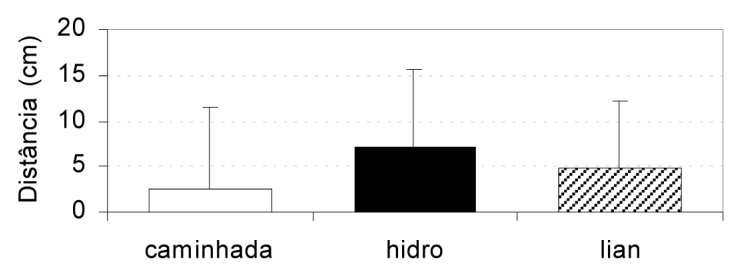

G
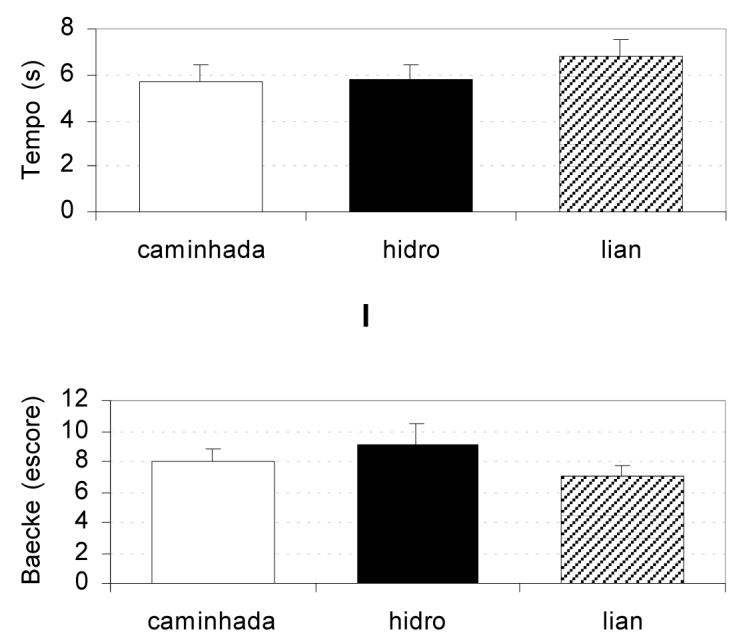

B

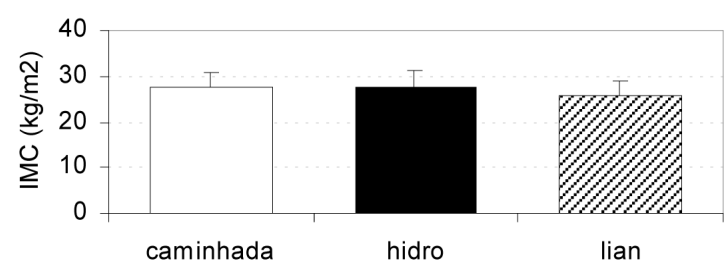

D

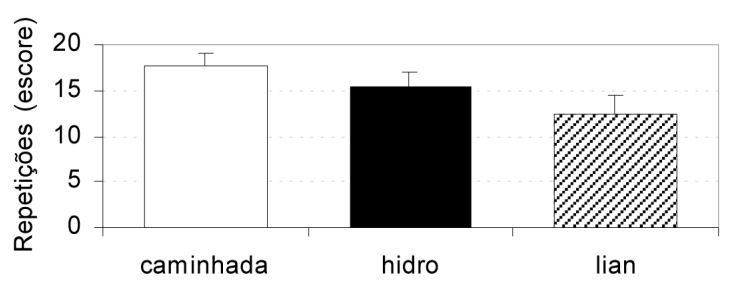

F

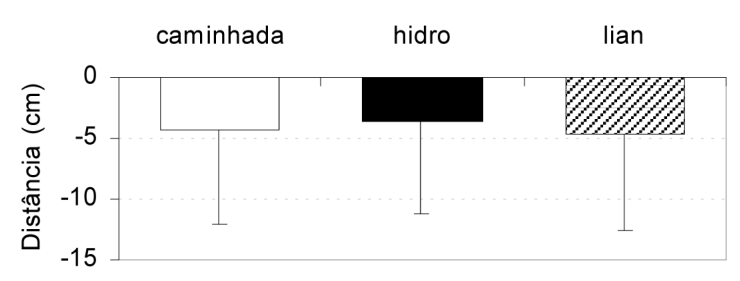

H

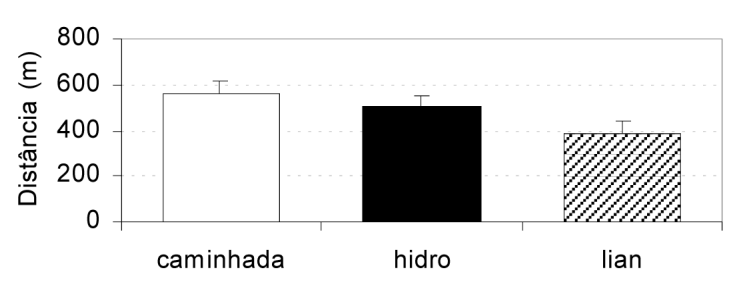

Figura 1. Média e desvios padrões da ldade $(\boldsymbol{A}), \operatorname{IMC}(\boldsymbol{B})$, Rosca (C), Levantar da Cadeira (D), Sentar e Alcançar $[\boldsymbol{E}$ ), Mãos nas Costas (F), Agilidade ( $\mathbf{G}$ ), Caminhar 6 minutos $(\boldsymbol{H})$ e Baecke (I) dos grupos Caminhada, Hidroginástica e Lian Gong. 
que foi adaptado e validado para ser utilizado com idosos como proposto por Voorrips et al.(24). O questionário consiste da soma das actividades de casa, actividades esportivas, actividades físicas diárias e de lazer, resultando em uma pontuação, classificando-as em um padrão alto, médio ou baixo de acordo com suas actividades diárias.

\section{Procedimentos}

Antes de iniciar a bateria de testes foi solicitado aos participantes que realizassem um aquecimento de cinco a dez minutos com movimentos de braços e pernas, alongamento de membros inferiores e superiores além de caminhadas curtas, com intuito de prepará-los para os testes. Todas as instruções a respeitos dos testes e avaliações foram fornecidas pelo mesmo instrutor.

\section{RESULTADOS}

O desempenho dos grupos Caminhada $(\mathrm{n}=38)$, Hidroginástica $(n=38)$, e Lian Gong $(n=37)$ referente à capacidade funcional e nível de actividade física foram comparados. A Figura 1 ilustra o desempenho dos três grupos e a Tabela 1 apresenta as médias e desvios padrões de cada variável estudada.

A ANOVA encontrou diferenças significantes para a idade dos participantes dos grupos estudados e o teste de Tukey localizou essa diferença entre o grupo Lian Gong e os grupos Caminhada $(\mathrm{p}<0,01)$ e Hidroginástica $(\mathrm{p}<0,05)$, ou seja, como pode ser visto na Figura 1(A), os participantes do grupo que pratica Lian Gong são mais velhos que os outros dois grupos.

Embora a ANOVA tenha encontrado diferença significante entre os grupos para o IMC

$(\mathrm{F}(2,110)=3,094, \mathrm{p}<0,001]$, o teste de Tukey não localizou tais diferenças $(\mathrm{p}>0,05)$. Entretanto, como podem ser observados na Tabela 1, os valores médios encontrados para o IMC dos três grupos, caminhada, hidroginástica e Lian Gong são respectivamente: 27,$40 ; 27,55$ e $25,83 \mathrm{~kg} / \mathrm{m} 2$. Portanto, é possível inferir que o grupo Lian Gong apresenta um IMC inferior quando comparado com os outros dois grupos.

Em relação aos testes que avaliam a força, ou seja, Rosca e Levantar da Cadeira, ilustrados na Figura 1(C) e 1(D), respectivamente, como não houve homogeneidade na variância (teste de Levene) foram utilizados testes não-paramétricos para essas variáveis. O teste Kruskal Wallis detectou diferença significante para força de membros inferiores (levantar da cadeira: $\left.\mathrm{x}^{2}=73,448, \mathrm{p}<0,001\right)$ e superiores (rosca: $\left.\mathrm{x}^{2}=29,692, \mathrm{p}<0,001\right)$. O teste $U$ de Mann-Whitney, indicou que o grupo Caminhada foi superior na força de membros inferiores quando comparado com o grupo Hidroginástica, que por sua vez é superior ao grupo Lian Gong. Entretanto no teste que avalia força de membros superiores (Rosca), o grupo Hidroginástica foi superior aos demais grupos, Caminhada e Lian Gong, que quando comparados entre si, não foram encontradas diferenças estatisticamente significantes.

Quando os grupos foram comparados através dos testes que avaliam a flexibilidade, tanto de membros inferiores, Sentar e Alcançar (Figura 1(E)], quanto de membros superiores, Mãos nas Costas [Figura 1 (F)], não foi observado diferença significante para ambos os testes [Sentar e alcançar: $\mathrm{F}(2,110)=2,788$, $\mathrm{p}>0,05$; e Mãos nas Costas: $\mathrm{F}(2,110)=0,229$, $\mathrm{p}>0,05]$. Portanto, os diferentes programas de actividade física avaliados se equivalem quando são comparados através do desempenho dessa capacidade motora.

A ANOVA encontrou diferença significante na comparação dos valores obtidos no teste de Levantar, Ir e Voltar $[F(2,110)=26,264, p<0,001]$. O teste de Tukey localizou essa diferença na comparação do grupo Lian Gong em relação aos grupos Caminhada e Hidroginástica $(p<0,001)$, indicando que o grupo Lian Gong mostrou desempenho inferior no que diz respeito à agilidade, levando mais tempo para completar a tarefa motora solicitada [Figura $1(\mathrm{G})$ ]. Como pode ser observado na Figura $1(\mathrm{H})$, no teste de Caminhar por 6 minutos, a ANOVA identificou diferença significante entre os grupos $[F(2,110)=96,818, p<0,001]$ e o Tukey $(p<0,001)$ detectou que, como poderia ser esperado, o grupo Caminhada foi superior aos outros dois grupos e o grupo Hidroginástica foi superior ao Lian Gong. $\mathrm{Na}$ avaliação do nível de actividade física diária que foi feita através do Questionário de Baecke como proposto por Voorrips et al.(24), a ANOVA encontrou diferença significante entre os grupos $[F(2,110)=30,929, \mathrm{p}<0,001]$ e o teste de Tukey 
Tabela 1. Médias e desvios padrões da idade [anos], IMC [ $\mathrm{kg} / \mathrm{m2}$ ], Questionário de Baecke (escore), e os testes Levantar da Cadeira (repetições], Rosca (repetições], Levantar, Ir e Voltar (segundos], Sentar e Alcançar (cm), Mãos nas Costas (cm) e Caminhar 6min (m).

\begin{tabular}{|c|c|c|c|c|c|c|c|c|c|}
\hline Grupos & Idade & IMC & Baecke & $\begin{array}{l}\text { Levantar } \\
\text { da Cadeira }\end{array}$ & Rosca & $\begin{array}{l}\text { Levantar, } \\
\text { Ir e Voltar }\end{array}$ & $\begin{array}{c}\text { Sentar } \\
\text { e Alcançar }\end{array}$ & $\begin{array}{l}\text { Mãos nas } \\
\text { Costas }\end{array}$ & $\begin{array}{l}\text { Caminhar } \\
6 \text { minutos }\end{array}$ \\
\hline \multicolumn{10}{|l|}{ Caminhada } \\
\hline Média & 66,05 & 27,40 & 7,94 & 17,65 & 16,07 & 5,67 & 2,57 & $-4,34$ & 559,28 \\
\hline Desvio padrão & 4,17 & 3,20 & 0,95 & 1,36 & 1,05 & 0,76 & 8,86 & 7,77 & 60,80 \\
\hline \multicolumn{10}{|l|}{ Hidroginástica } \\
\hline Média & 66,71 & 27,55 & 9,04 & 15,47 & 18,15 & 5,79 & 7,07 & $-3,65$ & 501,92 \\
\hline Desvio padrão & 3,74 & 3,72 & 1,41 & 1,43 & 1,55 & 0,61 & 8,68 & 7,63 & 49,69 \\
\hline \multicolumn{10}{|l|}{ Lian Gong } \\
\hline Média & 69,22 & 25,83 & 6,98 & 12,35 & 16,38 & 6,78 & 4,83 & $-4,74$ & 387,03 \\
\hline Desvio padrão & 4,96 & 2,95 & 0,77 & 2,04 & 3,40 & 0,80 & 7,27 & 7,84 & 52,37 \\
\hline
\end{tabular}

revelou que o grupo Hidroginástica é superior aos grupos Caminhada $(\mathrm{p}<0,001)$ e Lian Gong $(\mathrm{p}<0,001)$, e o grupo Caminhada é superior ao grupo Lian Gong $(\mathrm{p}<0,001)$ [Figura 1(I)]. Esse resultado indica que quando todas as actividades são computadas, o grupo Hidroginástica é o grupo mais activo.

\section{DISCUSSÃO}

O objectivo deste estudo foi realizar uma análise comparativa entre três programas de actividade física, Caminhada, Hidroginástica e Lian Gong, no sentido de identificar possíveis diferenças na capacidade funcional de seus praticantes. Parece razoável concluir que durante a caminhada há uma predominância do desenvolvimento da capacidade aeróbia, enquanto que, a hidroginástica, devido às propriedades físicas que o meio líquido oferece, possibilita o desenvolvimento de outras capacidades motoras como força e flexibilidade, além da própria capacidade aeróbia. Já o Lian Gong apresenta-se como uma alternativa de prática corporal de origem oriental que procura abordar o corpo como um todo, por meio de exercícios simples e objectivos que buscam resultados terapêuticos, através da melhora da circulação sanguínea melhorando a fisiologia das articulações e fortalecendo os músculos(17). Através da movimentação de membros superiores e inferiores característica dessa prática, é possível observar uma ênfase no desenvolvimento da capacidade motora flexibilidade. No sentido de detectar e mesmo analisar as diferenças entre os três programas estudados, foi utilizado um teste de capacidade funcional (Bateria de Testes de Fullerton) proposto por Rikli \& Jones(18,20), cujos autores propõem uma análise e classificação dos resultados a partir de uma sub-divisão por faixas etárias. Os intervalos de idade sugeridos foram 60-64, 65-69, 70-74, 75-79, 80-84, 85-89 e 90-94 anos de idade.

Embora a ANOVA tenha encontrado diferenças significantes para a idade dos participantes dos grupos estudados, ou seja, o grupo Lian Gong teve idade superior aos outros dois grupos, quando a média de idade do grupo é considerada, os mesmos fazem parte de um único grupo, já que a faixa etária sugerida pelos autores do teste é de 65 a 69 anos.

Nos testes que avaliam a flexibilidade de membros inferiores e superiores (Sentar e Alcançar e Mãos nas Costas), entretanto, os altos valores de desvio padrão encontrados demonstram maior heterogeneidade dos grupos estudados nesta capacidade motora. Apesar disso, todos os grupos encontram-se dentro da faixa de normalidade quanto à flexibilidade de membros inferiores e superiores, segundo a classificação de Rikli \& Jones(18,20). Os resultados sugerem que, embora os programas avaliados proporcionem um mesmo desempenho nos testes que avaliam flexibilidade, era esperado que o grupo Lian Gong obti- 
vesse performance superior aos outros dois grupos. Esse componente da aptidão física está entre as recomendações de atividade física para idosos do Colégio Americano de Medicina do Esporte(1). Tal recomendação se deve ao fato da observação de que exercícios de flexibilidade, não apenas aumentam a amplitude de movimento, mas também há evidências de que há uma diminuição de dores, em geral, além de trazer benefícios para as actividades de vida diária(1). A possível diminuição de dores que exercícios de flexibilidade proporcionam, seria coerente com a proposta terapêutica do Lian Gong.

A análise dos resultados referente ao teste Levantar da Cadeira, que avalia força de membros inferiores, mostrou que a Caminhada promove melhor desenvolvimento da força de membros inferiores quando comparado com a Hidroginástica e o Lian Gong. A prática do Lian Gong, porém, gerou um desempenho inferior. Entretanto quando comparamos com os dados da Rikli \& Jones ${ }^{(18,20)}$, o grupo Hidroginástica e o Lian Gong estão dentro da faixa de normalidade enquanto que a Caminhada esta acima da mesma faixa, ou seja, superior aos valores médios previstos para essa faixa etária sendo condizente com as afirmações de Lima ${ }^{(14)}$ que ressalta a Caminhada como um dos exercícios aeróbicos mais adequados para os adultos, especialmente idosos e indivíduos portadores de doenças cardíacas e metabólicas (diabetes, obesidade e excesso de triglicérides no sangue), produzindo os mesmos benefícios da corrida, em se tratando de efeitos aeróbios.

Quanto ao teste Levantar, Ir e Voltar que avalia agilidade e equilíbrio dinâmico, observou-se que o grupo Lian Gong teve desempenho inferior aos grupos Hidroginástica e Caminhada, ou seja, os indivíduos que Caminham ou fazem Hidroginástica são mais ágeis do que os indivíduos que praticam Lian Gong. Um aspecto que poderia ser considerado é o fato dos idosos que praticam Lian Gong serem, em média, quatro anos mais velhos.

A capacidade aeróbia dos grupos, medida pelo teste de Caminhar por 6 minutos, mostrou um resultado, de certa forma esperado, já que o grupo Caminhada teve o melhor desempenho, provavelmente pela especificidade do trabalho realizado, enquanto o grupo Lian Gong teve, novamente, desempenho inferior. A capacidade aeróbia é reduzida com o avanço da idade, limitando a intensidade e duração absoluta do exercício aeróbio sub-máximo(1,21). Entretanto, idosos não apresentam falência aparente do controle regulatório do sistema cardiovascular durante o exercício sub-máximo e demonstram as adaptações fisiológicas necessárias para o exercício aeróbio habitual(11). Sendo assim, a prática de actividades físicas que envolvam exercícios aeróbios merecem atenção dentro de um planejamento consciente. Shephard(21) ressalta a importância desse tipo de actividade para realizar actividades diárias como caminhar, ir ao shopping, ir a feira e participar de actividades recreativas ou esportivas.

Além da participação nos respectivos grupos de actividade física, propriamente dita, também foram avaliadas outras actividades que os participantes desse estudo, através do Questionário de Baecke(24). Nessa avaliação, o grupo Hidroginástica se mostrou mais activo em comparação aos outros grupos e o grupo Caminhada se mostrou mais activo em relação ao Lian Gong. Portanto, tais resultados indicam que o grupo de Hidroginástica tem maior gasto energético durante o seu dia-a-dia do que os participantes dos outros dois programas.

Em conjunto, os resultados desse estudo sugerem que os programas avaliados apresentam benefícios para a capacidade funcional e, consequentemente, à saúde de seus praticantes. Tais benefícios são identificados através da comparação dos resultados obtidos pelos grupos, particularmente, os grupos Caminhada e Hidroginástica, e as curvas de desempenho populacional de Rikli \& Jones $(18,20)$. Isto porque, em alguns testes, os idosos apresentaram desempenho superior à faixa de normalidade na capacidade motora avaliada, sugerindo que este desempenho talvez seja alcançado em função da participação no programa de atividade física.

As evidências que a actividade física regular é benéfica para indivíduos idosos são substanciais e, como afirmam Gass e Gass ${ }^{(10)}$, pode ser chamada de "Wonder Drug" para atenuar a degeneração associada com o avanço da idade. Entretanto, ainda não é claro qual é a dose que maximizará os benefícios relativos à saúde de idosos, ou quanto tempo é necessário para obter tais benefícios ${ }^{(10)}$. Essas informações são fundamentais para a obtenção de resultados eficientes do ponto de vista da saúde pública, no entanto, há de se valorizar preferências individuais na escolha da actividade física adequada. 


\section{CONCLUSÃO}

Dentro das limitações do presente estudo, pode-se concluir que na comparação dos programas de actividade física Caminhada, Hidroginástica e Lian Gong, foram observadas diferenças no desenvolvimento das capacidades motoras de idosos. Há indicativos de uma superioridade no desempenho dos grupos que praticam caminhada e hidroginástica que, além disso, poderiam ser consideradas como actividades complementares no desenvolvimento do conjunto das capacidades motoras de indivíduos idosos.

\section{AGRADECIMENTOS}

Este estudo foi apresentado no XI Congresso de Ciências do Desporto e Educação Física dos Países de Língua Portuguesa. Agradecemos as instituições onde foram realizadas as colectas de dados.

\section{REFERÊNCIAS BIBLIOGRÁFICAS}

1. ACSM (1998). Exercise and physical activity for older adults. Medicine Science Sports Exercise, 30(6): 992-1008

2. Baecke JAH, Burema J, Frijeters JER (1982). A short questionnaire for the measurement of habitual physical activity in epidemiological studies. American Journal Clinical Nutrition, 36: $936-942$

3. Baecke JAH, Burema J, Frijeters JER (1998). Baecke Questionnaire of habitual physical activity. Medicine Science Sports Exercise, 29(6, Supplement): s15-18

4. Blain H, Vuillemin A, Blain A (2000). The preventive effects of physical activity in the elderly. Presse Med, 29: 1240-1248

5. Bonachella V (1994). Manual básico de hidroginástica. Rio de Janeiro: Sprint.

6. Cech D, Martin S (1994). Functional movement development across the life span. $1^{\text {a }}$ edição, Elsevier Health Sciences: Saunders Company.

7. Chandler JM, Hadley EC (1996). Exercise to improve physiologic functional performance in old age. Clinics in Geriatric Medicine, 12: 761-784

8. Debert GG (1999). A reinvenção da velhice. São Paulo: FAPESP/EDUSP.

9. Fried LP, Bandeen-Roche K, Williamson JD, Prassada-Rao P, Chee E, Teppeer S, Rubin GS (1996). Functional decline in older adults: Expanding methods of ascertainment. Journal of Gerontology: Medical Sciences, 51A, M206-M214

10. Gass GC, Gass EM (2004). Is exercise the "Wonder Drug" for older individuals? European Review of Aging and Physical Activity, 1: 4-17

11. Gates PE, Moreau KL, Eskurza I, Seals DR (2005). Aging and cardiovascular function with acute and habitual exercise. European Review of Aging and Physical Activity, 2: 35-46

12. IBGE (2006). Censo demográfico 2000. Rio de Janeiro: IBGE. Disponível em <http://www.ibge.gov.br/>. Acesso em: Set 2006

\section{CORRESPONDÊNCIA}

\section{Marcelo Eduardo S. Nunes}

EEFE - USP

Laboratório de Comportamento Motor (Lacom)USP

Av. Prof. Mello Moraes, 65

CEP 05508-900-São Paulo - SP- BRASIL

E-mail: nunesme@globo.com
13. Kalache A (1996). Ageing worldwide. In: S. Ebrahim e A Kalache (Eds.), Epidemiology in old age. London: BMJ Publishing Group.

14. Lima DF (1998). Caminhadas: teoria e prática. Rio Janeiro, Sprint.

15. Marieke JG, Heuvelen V, Gertrudis IJM, Kempen JO, Piet R (1998). Physical fitness related to age and physical activity in older persons. Medicine Science Sports Exercise, 30: 3, 434441

16. Marques J, Pereira N (1999). Hidroginástica: exercícios comentados: cinesiologia aplicada à hidroginástica. Rio de Janeiro: Ney Pereira

17. Ming ZY (2001). Lian Gong Shi Ba Fa: ginástica terapêutica e preventiva. Editora Pensamento; $2^{a}$ edição 05-29

18. Rikli R, Jones J (1999a). Development and validation of a function fitness test for community-residing older adults. Journal of Aging and Physical Activity, 7: 129-161

19. Rikli R, Jones J (1999b). Functional fitness normative scores for community-residing older adults. Journal of Aging and Physical Activity, 7: 162-191

20. Rikli R, Jones J (2001). Senior Fitness Test Manual. Human Kinetics.

21. Shephard RJ (1997). Aging, physical activity, and health. Champaign, IL: Human Kinectics

22. Spirduso WW (1995). Physical dimensions of aging. Champaign, IL: Human Kinetics

23. Veras RP (1996). Ageing in developing countries: a case study of Brazil. In: S. Ebrahim e A. Kalache (Eds.), Epidemiology in old age. London: BMJ Publishing Group.

24. Voorrips LE, Ravelli ACJ, Dongelmans PCA, Deurenberg P, Staveren VWA (1991). A physical activity questionnaire for the elderly. Medicine Science Sports Exercise, 23: 974-979 